\title{
Modelling of non-stationary pressure fluctuations during boiling in a minichannel
}

\author{
Romuald Mosdorf*, Hubert Grzybowski, Iwona Gruszczyńska \\ Department of Mechanics and Applied Computer Science, Faculty of Mechanical Engineering, Bialystok University of Technology, \\ Wiejska 45 C, 15-351 Bialystok, Poland
}

\begin{abstract}
Boiling in a minichannel occurring at a low mass flow rate is accompanied by non-stationary twophase flow. The analysis of pressure fluctuations during non-stationary boiling in minichannel shows that quasi-periodic changes in flow patterns can be observed in such fluctuations. We can define in such a way the sequences, which are called "oscillating boiling patterns". In the present paper the model, which allows us to simulate the appearance of "oscillating boiling patterns" has been presented. In the proposed model the mass flow rate changes (because of evaporation and condensation) are modelled by compressible volumes representing various sizes bubbles. In the paper the good quality agreement between experimental data and simulation results has been achieved. Experimental data was collected during the boiling in open minichannels system with inner diameter of $1 \mathrm{~mm}$.
\end{abstract}

\section{Introduction}

Boiling in a minichannel occurring at a low mass flow rate is accompanied by non-stationary two-phase flow. Such flow forms a complex two phase flow pattern inside the minichannel. The chaotic void fraction and pressure drop fluctuations are observed. The flow patterns defined in stationary states such as: the bubble flow, the slug flow and the annular flow in non-stationary flow occur only in short time periods. The analysis of pressure fluctuations during non-stationary boiling in minichannel, presented in the paper [1], shows that quasi-periodic changes in flow patterns can be observed in such fluctuations. In short time periods there are repeated quasi-periodic sequences of flow patterns such as bubble flow, slug flow and annular flow. We can define in such a way the sequences, which are called "oscillating boiling patterns". In the experimental data the "oscillating boiling patterns" were identified using the RQA and PCA methods [2].

In the present paper the model, which allows us to simulate the appearance of "oscillating boiling patterns" has been presented. The model uses the concept of modelling the pressure drop oscillations proposed in the paper [1].

\section{Experimental setup}

The scheme of the experimental setup is shown in Fig. 1. The distance between minichannels was equal to $5 \mathrm{~mm}$ and inner diameter was equal to $1 \mathrm{~mm}$. Distilled water was supplied to the heated minichannels from individual surge tanks (Fig. $1-4,5$ ). The water flow rate was regulated by a ball valve (Fig. 1 - 2). The pressure inside the supply tank (Fig. 1 - 1) was kept constant with using the proportional pressure regulator. The heated section consists of two $150 \mathrm{~mm}$ long circular brass pipe with an inner diameter of $1 \mathrm{~mm}$. The two-phase flow patterns at the outlet of the heated section were observed in the glass tube (with an inner diameter of $1 \mathrm{~mm}$ ) and recorded by a high-speed camera (Fig. 1 - 8). The outlet of the glass tube was connected to the overflow tank (Fig. 1 - 13). In each minichannel the pressure drop was measured by silicon pressure drop sensor MPX12DP. Signals were acquired by data acquisition system at a rate of $1 \mathrm{kHz}$.

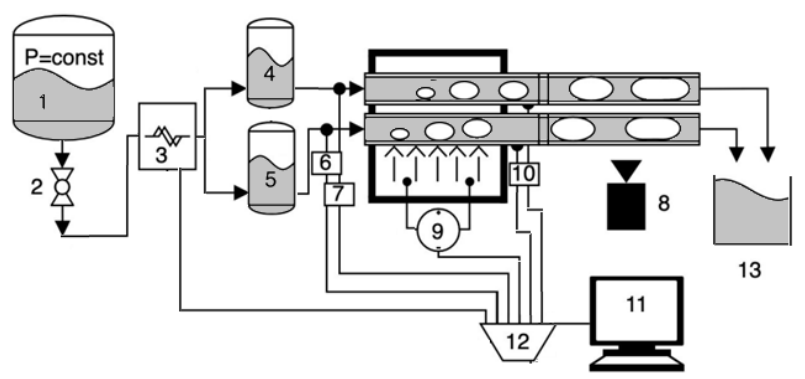

Fig. 1. Schematic diagram of the experimental setup. 1 - supply tank, 2 - ball valve, 3 - flow meter, 4,5 - surge tank, 6,7 - pressure sensor, 8 - high speed camera, 9 - power control, 10 - thermocouples, 11 - PC, 12 - data acquisition system, 13 - overflow tank.

The individual surge tanks were connected by pipe filled by water, it causes that pressure drop in one channel influences on the pressure changes in the second channel. Such a system prevents neighbouring channels from direct hydraulic interactions. Because of the relatively large distance between channels the thermal interaction between them appears with the long period of time.

\footnotetext{
* Corresponding author: r.mosdorf@pb.edu.pl
} 
Therefore we can conclude that in the system the major mechanism of channels interaction is pressure interactions which are associated with the pressure changes in surge tanks. Such interaction appears in a short period of time. The example of pressure drop oscillations is shown in Fig. 2. The long-period (approx. $300 \mathrm{~s}$ ) oscillations associated with the presence of compressible volume in the tested system were observed. Also, short-period density wave oscillations were superimposed on longperiod pressure drop oscillations.

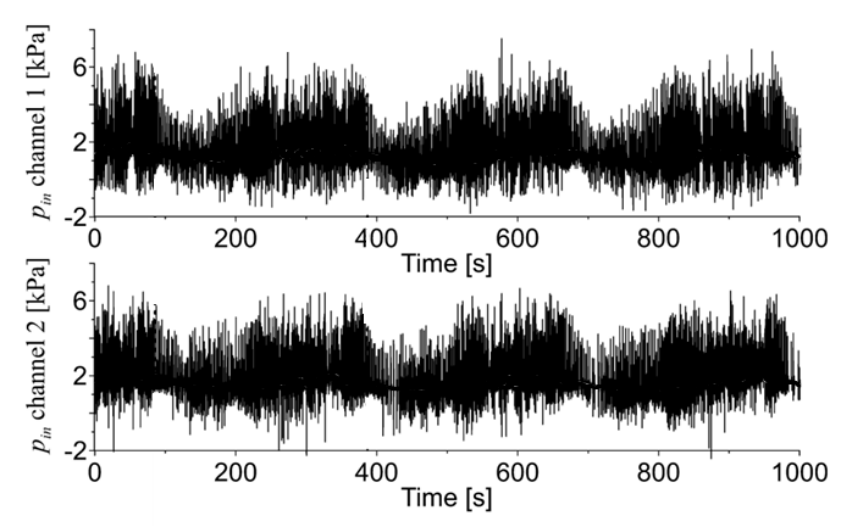

Fig. 2. The pressure drop oscillations at $G=38.8 \mathrm{~kg} / \mathrm{m}^{2} \mathrm{~s}$ and $P=55.8 \mathrm{~W}$.

\section{Modelling}

The system containing a minichannel and a compressible volume is considered. The pressure and mass flow rate fluctuations were modelled building equations of momentum and mass balance. A scheme of boiling system is shown in Fig. 3a. In the model considered in the work [1], it was assumed that the liquid flow rate on the beginning and the end of boiling minichannel are equal: $m_{o I}(t)=m_{\text {out }}(t)$. Data presented in the paper [2] show that both processes: liquid evaporation and vapour condensation (occurring outside and inside the heated mini-channel) lead to additional changes in liquid flow rate at the outlet of the minichannel, thus $m_{o l}(t) \neq m_{\text {out }}(t)$. Therefore, in the proposed model the mass flow rate changes (because of evaporation and condensation) are modelled by adding additional compressible volumes representing various sizes bubbles. The scheme of such a system is shown in Fig. 3b. In the part "I" of the system presented in Fig. 3b there is:

$$
\dot{m}_{\text {out }}^{1}=\dot{m}_{0}^{x 1}+\dot{m}_{b}^{1}
$$

where $\dot{m}_{b}^{1}$ - mass flow rate generated by bubble growth and its shrink during the condensation, $\dot{m}_{0}^{x 1}=x_{1} \dot{m}_{0}^{1}$, where $x_{1}+x_{2}=1$.

The Eq.(1) allows us to write the following equations:

$$
\begin{gathered}
\frac{d \dot{m}_{\text {out }}^{1}}{d t}=\frac{d \dot{m}_{0}^{x 1}}{d t}+\frac{d \dot{m}_{b}^{1}}{d t} \\
\frac{d^{2} \dot{m}_{\text {out }}^{1}}{d t^{2}}=\frac{d^{2} \dot{m}_{0}^{x 1}}{d t^{2}}+\frac{d^{2} \dot{m}_{b}^{1}}{d t^{2}}
\end{gathered}
$$

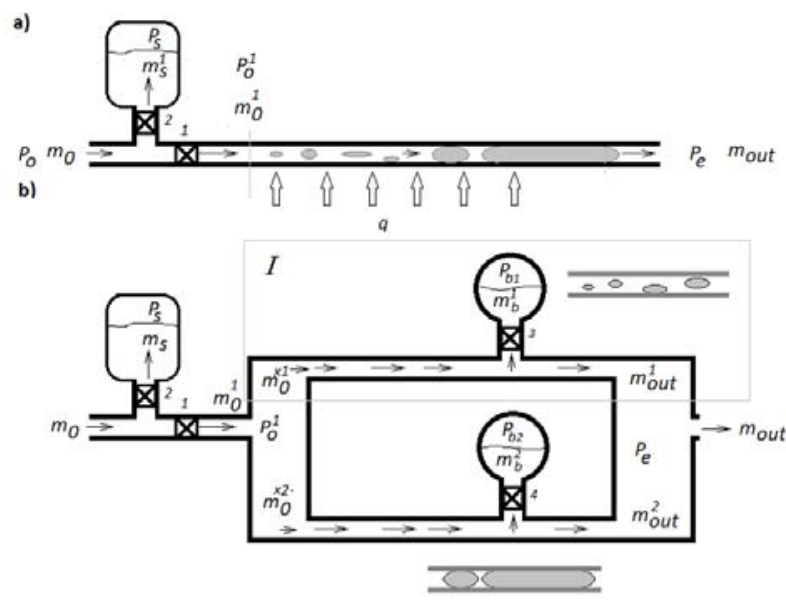

Fig. 3. The schemes of boiling systems with minichannel. a) system presented in the paper [1], b) present system.

For $\dot{m}_{b}^{1}$ the lumped momentum balance has a form:

$$
I_{s} \frac{d \dot{m}_{b}^{1}}{d t}=P_{e}-P_{b 1}-\Delta P_{r}^{3}
$$

where $I_{b}=\sum_{i} \frac{L_{i}}{A_{i}} ; \Delta P_{r}^{3}=\gamma_{3} \dot{m}_{b}^{1} ; \gamma_{3}$ - lumped restrictor model parameters.

The Eq.(4) allows us to write the following equation:

$$
I_{b} \frac{d^{2} \dot{m}_{b}^{1}}{d t^{2}}=\frac{d P_{e}}{d t}-\frac{d P_{b 1}}{d t}-\frac{d\left(\Delta P_{r}^{3}\right)}{d t}
$$

It was assumed that in the surge tank [1] $P_{b 1} V_{b 1}^{n}=$ constant, then:

$$
\frac{d P_{b 1}}{d t}=-\frac{n P_{b 1}}{V_{b 1}} \frac{d V_{b 1}}{d t}
$$

Because: $\frac{d V_{b 1}}{d t}=-\frac{\dot{m}_{b}^{1}}{\rho_{f}}$, then Eq.(6) has a following form:

$$
\frac{d P_{b 1}}{d t}=\frac{n P_{b 1}}{\rho_{f} V_{b 1}} \dot{m}_{b}^{1}
$$

Because $\dot{m}_{b}^{1}=\dot{m}_{\text {out }}^{1}-\dot{m}_{o}^{x 1}$, therefore Eq.(5) can be written as:

$$
\begin{gathered}
I_{b} \frac{d^{2} \dot{m}_{o u t}^{1}}{d t^{2}}-I_{b} \frac{d^{2} \dot{m}_{0}^{x 1}}{d t^{2}}=\frac{d P_{e}}{d t}-\gamma_{3} \frac{d \dot{m}_{o u t}^{1}}{d t}+ \\
\gamma_{3} \frac{d \dot{m}_{0}^{x 1}}{d t}-\frac{n P_{b 1}}{\rho_{f} V_{b 1}} \dot{m}_{o u t}^{1}+\frac{n P_{b 1}}{\rho_{f} V_{b 1}} \dot{m}_{0}^{x 1}
\end{gathered}
$$

The lumped momentum balance equation for flow in boiling channel has a form:

$$
I_{c} \frac{d \dot{m}_{\text {out }}^{1}}{d t}=P_{o}^{1}-P_{e}-\Delta P_{C}^{1}
$$

where : $\Delta P_{C}^{1}=\Delta P_{M C}^{1}$

$$
\begin{aligned}
& \Delta P_{M C}-\text { minichannel pressure drop } \\
& I_{C}=\sum_{i} \frac{L_{i}}{A_{i}}
\end{aligned}
$$

The Eq.(9) with constant $P_{e}$ allows us to write the following equation:

$$
I_{C} \frac{d^{2} \dot{m}_{o u t}^{1}}{d t^{2}}=\frac{d P_{O}^{1}}{d t}-\frac{d \Delta P_{C}^{1}}{d t}
$$


Adding the Eq.(10) and Eq.(8) with constant $P_{e}$ allows us to write the following equation:

$$
\begin{aligned}
& I I \frac{d^{2} \dot{m}_{\text {out }}^{1}}{d t^{2}}+\left[\frac{d\left(\Delta P_{C}^{1}\right)}{d \dot{m}_{\text {out }}^{1}}+\gamma_{3}\right] \frac{d \dot{m}_{\text {out }}^{1}}{d t}+ \\
& C_{s} \dot{m}_{\text {out }}^{1}=\frac{d P_{o}^{1}}{d t}+C_{s} \dot{m}_{0}^{x 1}+\gamma_{4} \frac{d \dot{m}_{0}^{x 1}}{d t}+I_{s} \frac{d^{2} \dot{m}_{0}^{x 1}}{d t^{2}} \\
& \text { where } C_{s}=\frac{n P_{b 1}}{\rho_{f} V_{b 1}} ; I=I_{c}+I_{b} .
\end{aligned}
$$

It has been assumed that $P_{o}^{1}=P_{0}-\Delta P_{r}^{1}$. Because $\Delta P_{r}^{2}=\gamma_{1} \dot{m}_{0}^{1}$ therefore $P_{o}^{1}=P_{0}-\gamma_{1} \dot{m}_{0}^{1}$, finally:

$$
\frac{d P_{0}^{1}}{d t}=\frac{d P_{0}}{d t}-\gamma_{1} \frac{d \dot{m}_{0}^{1}}{d t}=-\gamma_{1} \frac{d \dot{m}_{0}^{1}}{d t}=-\gamma_{1} x_{1} \frac{d \dot{m}_{0}^{x 1}}{d t}
$$

The Eq.(12) allows us to write the Eq.(11) in the following form:

$$
\begin{gathered}
I \frac{d^{2} \dot{m}_{o u t}^{1}}{d t^{2}}+\left[\frac{d\left(\Delta P_{C}^{1}\right)}{d \dot{m}_{o u t}^{1}}+\gamma_{3}\right] \frac{d \dot{m}_{o u t}^{1}}{d t}+C_{s} \dot{m}_{o u t}^{1}= \\
C_{S} \dot{m}_{0}^{x 1}+\left(\gamma_{3}-\gamma_{1} x_{1}\right) \frac{d \dot{m}_{0}^{\chi 1}}{d t}+I_{S} \frac{d^{2} \dot{m}_{0}^{x 1}}{d t^{2}}
\end{gathered}
$$

It has been assumed that [1]:

$$
\frac{d \Delta P_{C}^{1}}{d \dot{m}_{o u t}^{1}}=\frac{d \Delta P_{M C}^{1}}{d \dot{m}_{o u t}^{1}}=\delta_{1}\left(\dot{m}_{o u t}^{1}-\dot{m}_{a}\right)\left(\dot{m}_{o u t}^{1}-\dot{m}_{b}\right)
$$

Inserting Eq.(14) to Eq.(13), it was obtained:

$$
\begin{aligned}
& \frac{d^{2} \dot{m}_{\text {out }}^{1}}{d t^{2}}+\frac{1}{I}\left[\delta_{1}\left(\dot{m}_{\text {out }}^{1}-\dot{m}_{a}\right)\left(\dot{m}_{\text {out }}^{1}-\dot{m}_{b}\right)+\gamma_{3}\right] \frac{d \dot{m}_{\text {out }}^{1}}{d t}+ \\
& \frac{C_{s}}{I}\left(\dot{m}_{\text {out }}^{1}-\dot{m}_{o}^{x 1}\right)=\frac{\left(\gamma_{3}-\gamma_{1} x_{1}\right)}{I} \frac{d \dot{m}_{0}^{x 1}}{d t}+\frac{I_{S}}{l} \frac{d^{2} \dot{m}_{0}^{x 1}}{d t^{2}}
\end{aligned}
$$

Assuming that [1]: $Z_{0}^{1}=\dot{m}_{o}^{x 1}, Z^{1}=\dot{m}_{\text {out }}^{1}-Z_{0}^{1}, Z_{a}^{1}=$ $\dot{m}_{0}^{x 1}-\dot{m}_{a}, Z_{b}^{1}=\dot{m}_{0}^{x 1}-\dot{m}_{b}$. it was obtained:

$$
\begin{gathered}
\frac{d \dot{m}_{o u t}^{1}}{d t}=\frac{d Z^{1}}{d t}+\frac{d \dot{m}_{O}^{\chi 1}}{d t} \\
\frac{d^{2} \dot{m}_{\text {out }}^{1}}{d t^{2}}=\frac{d^{2} z^{1}}{d t^{2}}+\frac{d^{2} \dot{m}_{o}^{\chi 1}}{d t^{2}}
\end{gathered}
$$

Equation (15) was written in the form:

$$
\begin{array}{r}
\frac{d^{2} Z^{1}}{d t^{2}}+\left[a\left(Z^{1}\right)^{2}+b Z^{1}+c\right]\left(\frac{d Z^{1}}{d t}+\frac{d \dot{m}_{O}^{\chi 1}}{d t}\right)+ \\
\frac{C_{S}}{I}\left(Z^{1}\right)=\frac{\left(\gamma_{3}-\gamma_{1} x_{1}\right)}{I} \frac{d \dot{m}_{0}^{\chi 1}}{d t}+\left(\frac{I_{S}}{I}-1\right) \frac{d^{2} \dot{m}_{0}^{\chi 1}}{d t^{2}}
\end{array}
$$

When $\dot{m}_{0}^{x 1}$ is constant, then Eq.(17) has a form considered in the paper [1]:

$$
\frac{d^{2} Z^{1}}{d t^{2}}+\left[a\left(Z^{1}\right)^{2}+b Z^{1}+c\right]\left(\frac{d Z^{1}}{d t}\right)+\frac{C_{s}}{I}\left(Z^{1}\right)=0
$$

The Eq.(18) was used for modeling the pressure oscillations, when $m_{o}=$ constant [1]. The pressure drop can be calculated by integration of [1] $a\left(Z^{1}\right)^{2}+b Z^{1}+$ $c-\gamma_{3}$, therefore:

$$
\Delta P_{M C}=\frac{a}{3}\left(Z^{1}\right)^{3}+\frac{b}{2}\left(Z^{1}\right)^{2}+c\left(Z^{1}\right)-\gamma_{3}\left(Z^{1}\right)+e
$$

where $e$ - constant

In non-stationary pressure fluctuations the pressure drop is described by the following equation [1]:

$$
\begin{gathered}
\Delta P_{\text {channels }}=\Delta P_{M C}+I_{b} \frac{d Z^{1}}{d t}=\frac{a}{3}\left(Z^{1}\right)^{3}+ \\
\frac{b}{2}\left(Z^{1}\right)^{2}+c\left(Z^{1}\right)-\gamma_{3}\left(Z^{1}\right)+e+I_{b} \frac{d Z^{1}}{d t}
\end{gathered}
$$

The set of differential equations describing the mass flow rates in the system (Fig. 3b) is as follows:

$$
\begin{gathered}
\frac{d^{2} G}{d t^{2}}+\left[a(G)^{2}+b G+c\right]\left(\frac{d G}{d t}\right)+\frac{C_{s}}{I}(G)=0 \\
\frac{d^{2} Z^{1}}{d t^{2}}+\left[\delta_{1}\left(Z^{1}-\dot{m}_{a}\right)\left(Z^{1}-\dot{m}_{b}\right)+\gamma_{3}\right]\left(\frac{d Z^{1}}{d t}+\frac{d G_{1}}{d t}\right)+ \\
\frac{C_{s}}{I}\left(Z^{1}\right)=\frac{\left(\gamma_{3}-\gamma_{1} x_{1}\right)}{I} \frac{d G_{1}}{d t}+\left(\frac{I_{s}}{I}-1\right) \frac{d^{2} G_{1}}{d t^{2}} \\
\frac{d^{2} Z^{2}}{d t^{2}}+\left[\delta_{2}\left(Z^{2}-\dot{m}_{a}\right)\left(Z^{2}-\dot{m}_{b}\right)+\gamma_{3}\right]\left(\frac{d Z^{2}}{d t}+\frac{d G_{2}}{d t}\right)+ \\
\frac{C_{S}}{I}\left(Z^{2}\right)=\frac{\left(\gamma_{4}-\gamma_{1} x_{2}\right)}{I} \frac{d G_{2}}{d t}+\left(\frac{I_{S}}{I}-1\right) \frac{d^{2} G_{2}}{d t^{2}}
\end{gathered}
$$

where:

$$
\begin{gathered}
G_{1}=x_{1} G ; G_{2}=x_{2} G ; x_{1}+x_{2}=1 \\
G_{0}=m_{0}, G=\dot{m}_{o}^{1}-G_{0} \\
\dot{m}_{o}^{1}=G+G_{0} \\
Z_{0}^{1}=\dot{m}_{o}^{x 1}, Z^{1}=\dot{m}_{\text {out }}^{1}-\dot{m}_{o}^{x 1}, \\
\dot{m}_{\text {out }}^{1}=Z^{1}+\dot{m}_{o}^{x 1} \dot{m}_{\text {out }}^{1}=Z^{1}+\dot{m}_{o}^{x 1} \\
Z_{0}^{2}=\dot{m}_{o}^{x 2}, Z^{2}=\dot{m}_{\text {out }}^{2}-\dot{m}_{o}^{x 2}, \\
\dot{m}_{\text {out }}^{2}=Z^{2}+\dot{m}_{o}^{x 2} \dot{m}_{\text {out }}^{2}=Z^{2}+\dot{m}_{o}^{x 2}
\end{gathered}
$$

In Fig. 4 it is shown the example of mass flow rate changes in time of 100 seconds. The red line indicates the mean value of liquid flow rate during the moving $2 \mathrm{~s}$ period.

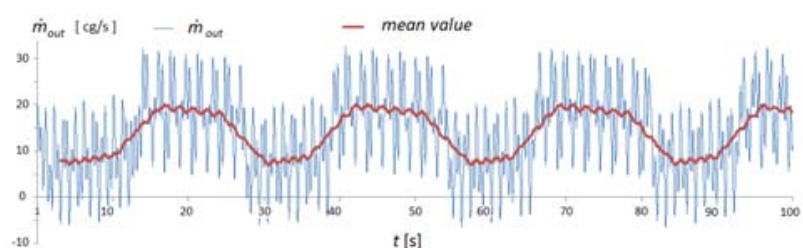

Fig. 4. The example of mass flow rate changes in time of 100 seconds. The red line indicates the mean value of liquid flow rate during the moving $2 \mathrm{~s}$ period. $\delta_{l}=\delta_{2}=1.64, m_{a}=-4[\mathrm{cg} / \mathrm{s}], m_{b}=2.9$ $[\mathrm{cg} / \mathrm{s}], \quad m_{0}=16[\mathrm{cg} / \mathrm{s}], \quad a=1.4283, \quad b=1.6163, \quad c=-14.4716$, $d=1.0028, I_{1}=0.0530, I_{2}=0.530, \gamma_{l}=\gamma_{2}=0.3719, \gamma_{3}=\gamma_{4}=0.3719 / 2$, $e=10.9159, I_{b 1}=0.0447, I_{b 2}=0.447$.

\section{Data analysis and conclusion}

The recurrence plot (RP) method [3,4] is a tool that was introduced for visualization of the recurrence occurring in a chaotic dynamical system. The preparation of the RP starts from the attractor reconstruction from time series $x_{i}$. The attractor reconstruction in a $\mathrm{m}$ dimension space is carried out using the stroboscope coordination [5]. In this method the subsequent coordinates of attractor points $\left(X_{i}\right)$ are calculated based on 
the subsequent samples, between which the distance is equal to time delay $\tau$. The time delay is a multiplication of time between the samples. The mutual information method [6,7] is the most commonly used method for determining the proper delay time $\tau$ for the reconstruction of the attractor. The first minimum of the mutual information function is the proper value of $m$. The false nearest neighbours algorithm [8] is used to determine the proper embedding dimension $\mathrm{m}$ of the attractor in the phase space.

The points arrangement on the RP characterizes the properties of the dynamic system [4]. Zbilut and Webber [3] proposed a method called Recurrence Quantification Analysis (RQA) to determine the coefficients characterizing the RP. These indicators have been described in detail in [3]. A window RQA was carried out using a window with of $5 \mathrm{~s}$. The example of RP of mass flow rate changes in time is presented in Fig. 5.

a)

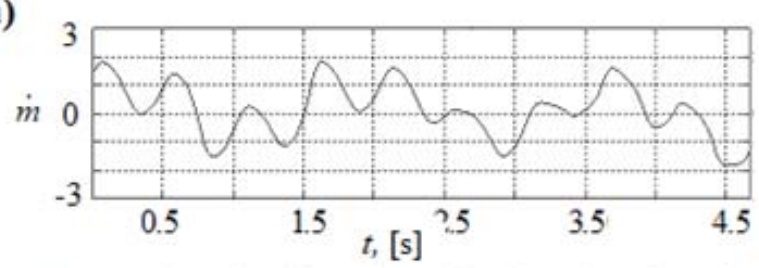

b)

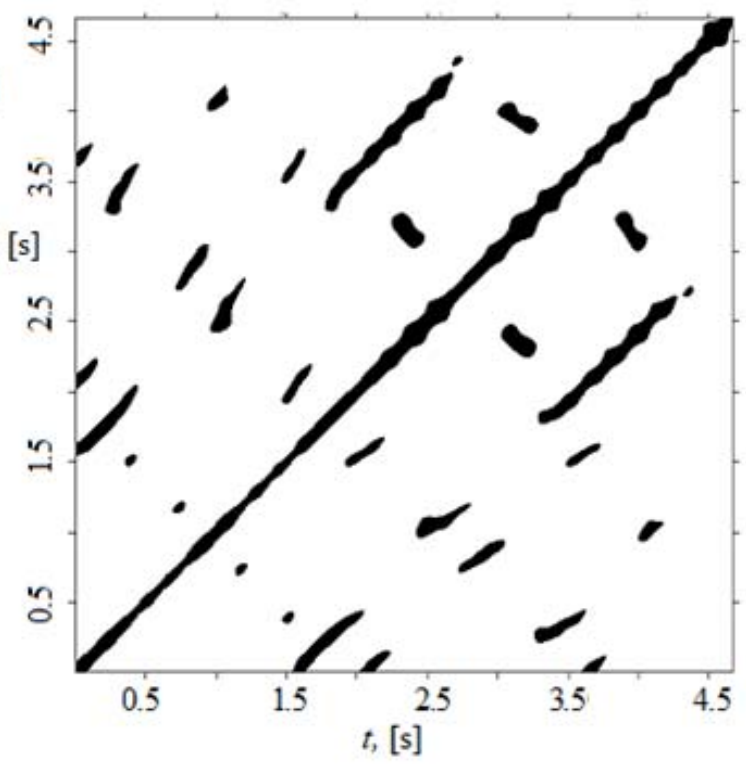

Fig. 5. The example of RP of mass flow rate changes in time.

The principal component analysis (PCA) is a method that uses the orthogonal transformation to convert an input set of data (in our case it is RQA coefficients) into a new set of data, in a new set of coordinates called the principal components. This transformation is defined in such a way that the data (in a new set of coordinates) has the largest possible variance around the first principal component. For the successive components the variance of data decreases.

Fig. 6 shows the results of the PCA analysis of the time series shown in Fig. 4.

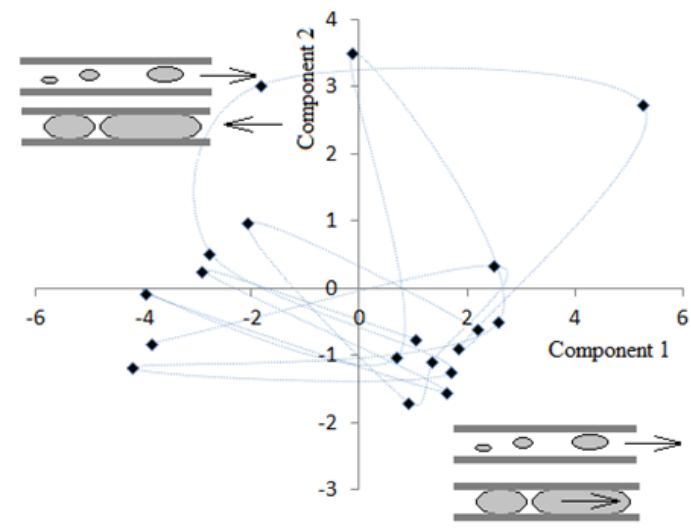

Fig. 6. The results of the PCA analysis of the time series shown in Fig. 4.

The negative values of the first component occur in intervals in which a significant change in the mean flow rate occurs.

In Fig. 7 there are presented the results of using the RQA and PCA methods for "oscillating boiling patterns" identification. The third component of PCA identifies the different kinds of mass flow rate oscillations.

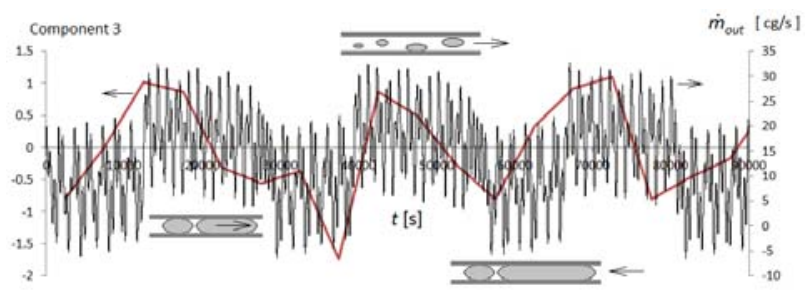

Fig. 7. Identification of different kinds of mass flow rate oscillations using the third component of PCA.

\section{Conclusions}

In the paper the good quality agreement between experimental data and simulation results has been achieved.

The change of two-phase flow pattern in minichannels system is accompanied by a change in the volume of vapour bubbles/slugs, which causes the change of compressible volume in the two-phase flow system. This leads to appearance of unending oscillations of void fraction with a frequency determined by the current volume of compressible volume (characteristic for a given flow pattern). The results of numerical simulations show that such oscillations can be interpreted as "oscillating boiling patterns", i.e. appearance of specific sequence of two-phase flow patterns which are accompanied by pressure drop fluctuations occurring at different frequencies (determined by varying volume of compressible space).

\section{Acknowledgments}

The study has been accomplished under the research project UMO-2017/27/B/ST8/02905 financed by the Polish National Science Centre. 


\section{References}

1. T. Zhang, Y. Peles, J. T. Wen, T. Tong, J. Y. Chang, R. Prasher, M. K. Jensen, Int. J. Heat Mass Transf. 53, 2347-2360 (2010)

2. H, Grzybowski, R. Mosdorf R. Int. J. Heat Mass Transf. 95, 25-32 (2018)

3. J.P. Zbilut, C.L. Webber, Phys. Lett. A. 171, 199-203 (1992)

4. N. Marwan, M. Carmen Romano, M. Thiel, J. Kurths, Phys. Rep. 438, 237-329 (2007)

5. H.G. Schuster, third edition, Wiley-VCH, Weinheim. (1995)

6. E. Bradley, H. Kantz, Chaos 25(9): 097610 (2015)

7. H. Kantz H., T. Schreiber, Cambridge University Press 7, (2004)

8. M.B. Kennel, R. Brown, H.D.I. Abarbanel, Phys. Rev. A. 45, 3403-3411 (1992) 